\title{
Rhipicephalus microplus and Ixodes ovatus cystatins in tick blood digestion and evasion of host immune response
}

Luís Fernando Parizi ${ }^{1 \dagger}$, Gabriela Alves Sabadin ${ }^{1+}$, María Fernanda Alzugaray ${ }^{1,3}$, Adriana Seixas ${ }^{1,4,7}$, Carlos Logullo ${ }^{5,7}$, Satoru Konnai ${ }^{2}$, Kazuhiko Ohashi ${ }^{2}$, Aoi Masuda ${ }^{1,7}$ and Itabajara da Silva Vaz $\mathrm{Jr}^{1,6,7^{*}}$

\begin{abstract}
Background: Cystatins are a group of cysteine protease inhibitors responsible for physiological proteolysis regulation and present in a wide range of organisms. Studies about this class of inhibitors in parasites have contributed to clarify their roles in important physiological processes, like blood digestion and modulation of host immune response during blood feeding. Thus, cystatins are a subject of research on the development of new parasite control methods. Additionally, the characterization of proteins shared by different parasite species represents a valuable strategy to find potential targets in multi-species control methods. However, cystatin functions in ticks remain undetermined, especially in Rhipicephalus microplus and Ixodes ovatus, two species that affect livestock and human health, respectively.
\end{abstract}

Methods: Here we report the inhibitory profile of two R. microplus (BrBmcys2b and BrBmcys2c) and one I. ovatus (Jplocys2a) cystatins to commercial cathepsins B, C, and L. The presence of native cystatins in R. microplus tissues was analyzed using sera against recombinant BrBmcys $2 b$ and BrBmcys2c. Also, a peptide from Jplocys2a was synthesized for rabbit immunization, and this serum was used to analyze the cross antigenicity between $R$. microplus and I. ovatus cystatins.

Results: Enzymatic inhibition profile of tick cystatins shows a distinct modulation for cathepsins related to tick blood digestion and evasion of host immune response. Furthermore, BrBmcys $2 \mathrm{~b}$ was detected in saliva and different tissues along tick stages, while BrBmcys2c was detected mainly in gut from partially engorged $R$. microplus females, demonstrating a distinct pattern of cystatin expression, secretion and traffic between tick tissues. Moreover, phylogenetic analysis suggests that Jplocys2a belongs to the group of tick gut secreted cystatins. Finally, cross-antigenicity assays revealed that antibodies against the Jplocys2a peptide recognize native and recombinant $R$. microplus cystatins.

Conclusion: The presence of these proteins in different tissues and their ability to differentially inhibit cathepsins suggest distinct roles for Jplocys2a, BrBmcys2b, and BrBmcys2c in blood digestion, egg and larvae development, and modulation of host immune response in tick physiology. The cross-antigenicity between native and recombinant cystatins supports further experiments using Jplocys2a, BrBmcys2b, and BrBmcys2c as vaccine antigens.

Keywords: Inhibitor, Cystatin, Tick, Rhipicephalus microplus, Ixodes ovatus

\footnotetext{
*Correspondence: itabajara.vaz@ufrgs.br

${ }^{\dagger}$ Equal contributors

'Centro de Biotecnologia, Universidade Federal do Rio Grande do Sul,

Avenida Bento Gonçalves, 9500, Prédio 43421, Porto Alegre 91501-970, RS, Brazil

${ }^{6}$ Faculdade de Veterinária, Universidade Federal do Rio Grande do Sul,

Avenida Bento Gonçalves, 9090, Porto Alegre 91540-000, RS, Brazil

Full list of author information is available at the end of the article
}

\section{Biomed Central}

(c) 2015 Parizi et al.; licensee BioMed Central. This is an Open Access article distributed under the terms of the Creative Commons Attribution License (http://creativecommons.org/licenses/by/4.0) which permits unrestricted use, distribution, and reproduction in any medium, provided the original work is properly credited. The Creative Commons Public Domain Dedication waiver (http://creativecommons.org/publicdomain/zero/1.0/) applies to the data made available in this article, unless otherwise stated. 


\section{Background}

Cystatins are tightly binding inhibitors of cysteine proteases [1]. In parasites, these inhibitors are involved in internal protective and regulatory biological processes [2] as well as in the modulation of the host's defense responses [3]. Classically, cystatins are divided into three groups, known as stefin (type 1), cystatin (type 2), and kininogen (type 3), although in ticks only stefins and cystatins have been reported [4]. It was only in recent years that the physiological functions of tick cystatins $[4,5]$ and their target cysteine cathepsins [6,7] during tick blood-feeding, digestion and development became the object of more consistent research. Previous studies have also demonstrated that tick cystatins modulate host cathepsins involved in processes like inflammation, antigen processing and presentation, phagocytosis, and cytokine expression [2]. The regulation of these physiological processes by tick cystatins promotes blood uptake and survival of parasite while it is attached to the host.

The first tick cystatin biochemically or molecularly characterized was isolated from Amblyomma americanum [8]. Back then, the participation of this cystatin was implicated in host immunomodulation and tick protection to harmful ingested factors during blood feeding. In Ixodes scapularis, two cystatins were biochemically characterized, Sialostatin L and Sialostatin L2 $[9,10]$. Sialostatin L showed host immune system modulation and cathepsins L, V, C, X, B, and papain inhibition, while Sialostatin L2 inhibited cathepsins L, V, S, and C, showing higher expression rate in late feeding. The enzymatic inhibition profile of Haemaphysalis longicornis cystatins for papain, cathepsins L, B, H, as well as tick cathepsins have been characterized in previous research [7,11-14]. Furthermore, it was demonstrated that some of the cystatins from $H$. longicornis play a role in innate immunity [11] and blood feeding [7,14]. Cystatins from Ornithodoros moubata were able to inhibit cathepsins B, $\mathrm{L}, \mathrm{S}, \mathrm{H}$, and $\mathrm{C}[15,16]$, and affect T-cell and dendritic cells proliferation and cytokine release [16]. Additionally, it was suggested that one Rhipicephalus appendiculatus cystatin present in nymph, male and female gut after feeding is involved in blood digestion process [17]. Taken together, these results indicate that cystatins play widespread and distinct regulatory roles in different tick species.

Rhipicephalus microplus is one of the most consistently studied cattle tick species, mainly because of the potentially expressive economic losses it causes in the livestock industry [18]. However, despite the great interest in understanding the physiology of this tick, few studies have analyzed $R$. microplus cystatins. Some $R$. microplus cysteine proteases were identified and characterized [19-21], demonstrating the importance of these enzymes in a variety of physiological processes and parasite stages. Nevertheless, few $R$. microplus cystatins and its target cysteine proteases have been characterized, including only one type 2 cystatin [5], named Rmcystatin3. Rmcystatin-3 is expressed in tick fat body, salivary glands, and hemocyte, though it inhibits cathepsin L, B, and $\mathrm{BmCl}-1$, a gut $R$. microplus cysteine endopeptidase [19], which suggests its role in tick blood digestion. Consequently, the control of $R$. microplus cysteine proteases activities by cystatins remains essentially unknown. In a previous work [22] we analyzed the sequence properties and immunogenicity of putative cystatins from $R$. microplus. These cystatins showed a high degree of homology among Rhipicephalus spp., differential RNA expression patterns in tick tissues, as well as cross-reactivity between them, suggesting the existence of shared epitopes.

The tick Ixodes ovatus geographic prevalence has been reported in Southeast Asia countries [23,24]. Its main hosts are humans, and bite cases have been observed in Tibet, Burma, Nepal, Japan, and China [25]. Borrelia burgdorferi and Ehrlichia species, which are the causal agents of Lyme disease and ehrlichiosis, respectively, are transmitted by ixodid ticks, and I. ovatus was found to be infected with Borrelia and Ehrlichia species [26-28]. However, no human cases of Lyme disease and ehrlichiosis transmitted by $I$. ovatus have been confirmed to date [27]. Also, no cystatins were characterized for this tick species so far.

Tick control is a great challenge in livestock and public health management worldwide, and relies on the use of synthetic acaricides [29]. In spite of that, vaccines have emerged as an interesting alternative method to decrease tick populations and the incidence of tickborne diseases in the environment [30]. Since hosts in several regions are exposed to multi-tick infestation, the development of a single vaccine against multiple species may be advantageous in control strategies against these parasites. A number of vaccination experiments showed the potential use of tick proteins to protect hosts against more than one tick species, demonstrating the feasibility of induction of cross-protection [31]. These tick protective proteins are present in many physiological processes, like Bm86, a gut protein of unknown function [32]; glutathione-S transferase, an enzyme responsible to detoxification of cell xenobiotic compounds [33]; ferritins, iron-storage proteins [34]; a cement protein named 64TRP [35]; and subolesin, a gene-expression regulator [36]. In fact, some of these tick antigens, such as Bm86 and its homologues, developed a higher protective host immune response to different tick species, rather than the tick species from which the antigen was isolated [31]. Vaccination trials using cystatins as antigens were performed against I. scapularis [37] and Ornithodoros moubata [16] infestations, showing the potential of these inhibitors to compose a vaccine against ticks. Despite the importance of cross antigenicity analysis as a 
preliminary step to detect multi-species antigen candidates, the potential of cross-protection induced by tick cystatins has yet to be analyzed.

In order to improve the understanding of the physiological roles of cystatins and the potential of these inhibitors as antigen in a multi-species vaccine, we characterized the inhibitory profile, tissue expression, and cross-antigenicity of a new I. ovatus cystatin, JpIocys2a, and two $R$. microplus cystatins, BrBmcys $2 \mathrm{~b}$ and BrBmcys2c. The presence of these proteins in different tick tissues and their ability to differently inhibit cathepsins suggest distinct roles for JpIocys2a, BrBmcys2b, and BrBmcys2c in blood digestion and modulation of host immune response in tick physiology. The crossantigenicity among cystatins from these two tick species paves the way for further experiments using JpIocys2a, BrBmcys $2 b$, and BrBmcys2c as vaccine antigens.

\section{Methods}

\section{Animals and ticks}

Partially and fully engorged female ticks (Porto Alegre $R$. microplus strain) were collected from Hereford (Bos taurus taurus) cattle for tissue dissection. Partially engorged $R$. microplus females weighing between 25 and $60 \mathrm{mg}$ were recovered manually from calves [38]. New Zealand White rabbits and Hereford cattle were housed at the Faculdade de Veterinária of the Universidade Federal do Rio Grande do Sul, Brazil. The experiments were approved and conducted following the guidelines of the Ethics Committee on Animal Experimentation of the same university. I. ovatus adult ticks were collected by flagging from the lower vegetation in forests in Hokkaido, Japan, and maintained by experimental infestation on hamsters to full engorgement. Hamsters were maintained in a P3 animal facility at Graduate School of Veterinary Medicine, Hokkaido University in accordance with the Institutional Animal Care and Use Committee guidelines.

\section{Cloning of cystatin ORF sequences}

For cloning of the JpIocys $2 a$ sequence, primers 5 '-GACTA GTCGCCAGCACGATGGCT-3' (forward) and 5' ${ }^{\prime}$-TGTCA TTTAACATGCGGCTGACGTC-3' (reverse) were designed based on TIGR nucleotide database (TC51659) from I. scapularis gene sequence with high similarity to cystatins. One ORF was amplified by RT-PCR from $I$. ovatus ovary RNA and cloned in pGEM-T vector (Promega). For cloning the DNA sequence encoding the mature JpIocys2a protein in the expression vector, the plasmid pGEM-T-JpIocys2a was amplified using the primers 5' -TTTTTGGATCCGGGTCGGCGAGCAGG TC-3' (forward) and 5' -AAAAAGAATTCCTAGACATTA TTAGGAGCTTCGCAGTGGTAG-3' (reverse). The PCR product was hydrolyzed with Bam $\mathrm{HI}$ and Eco RI
(Invitrogen) restriction enzymes and separated by electrophoresis on $0.8 \%$ agarose gel. The 396-bp fragment was excised, purified using the Geneclean II Kit (Qbiogene), and ligated into plasmid pGEX-4 T-1 (GE Healthcare) downstream the Glutathione S-transferase (GST) gene, which codifies the fusion protein. The plasmid pGEX-4T-1-JpIocys2a was transformed into Escherichia coli XL1BLUE strain. Transformed cells were plated and cultivated in LB agar containing ampicillin and chloramphenicol $(50 \mu \mathrm{g} / \mathrm{mL})$. The plasmid was purified and the cloned product was confirmed by sequencing.

The BrBmcys2b and BrBmcys2c nucleotide sequences were previously cloned [22]. In short, cystatin-coding regions were amplified by PCR from $R$. microplus salivary gland cDNA and cloned into plasmid pET-5a (Novagen).

\section{In silico analyses}

JpIocys2a DNA sequence analysis, amino acid predictions, and sequences alignment were conducted using the BioEdit version 7.2.5 software [39]. The cystatin antigenic index was calculated using the Jameson-Wolf algorithm in the software LASERGENE, version 7.0.0, to predict antigenic determinants by combining existing methods for protein structural predictions [40]. For phylogenetic analysis, an unrooted neighbor-joining phylogenetic tree was created using the MEGA software, version 5 [41]. Bootstrap support was assessed using 1000 replicates. The GenBank accession numbers for cystatins used in analysis were: $R$. microplus BrBmcys $2 \mathrm{~b}$ [GenBank: KC816580], R. microplus BrBmcys2c [GenBank: KC816581], R. microplus Rmcystatin-3 [GenBank: AIX97454]; I. ovatus JpIocys2a [GenBank: KP253747]; I. scapularis sialostatin 1 [GenBank: AF483724], I. scapularis sialostatin 2 [GenBank: DQ066048]; H. longicornis Hlcyst-2 [GenBank: DQ364159], H. longicornis Hlcyst-3 [GenBank: EU426545].

\section{Peptide synthesis}

To raise antibodies against conserved and exposed regions among tick cystatins, a peptide with 14 amino acids from the Jplocys2a amino acid sequence was synthesized (STQpep). Antigenic analysis of cystatins and percentage of identity among JpIocys2a, BrBmcys2a, BrBmcys2b, Brbmcys2c, and other tick cystatins were used to select the peptide. Peptides were kindly provided by Maria Aparecida Juliano, Department of Biophysics, Federal University of São Paulo (UNIFESP), SP, Brazil. An automated bench-top simultaneous multiple solidphase peptide synthesizer (PSSM 8 system, Shimadzu) was used for the solid-phase synthesis of the peptides by the Fmoc-procedure. Final peptides were deprotected in trifluoracetic acid and purified by semipreparative HPLC using an Econosil C-18 column. Analytical HPLC was performed using a binary HPLC system from Shimadzu 
with a SPD-10AV Shimadzu UV-vis detector, coupled to an Ultrasphere C-18 column. The HPLC column elutes were monitored by their absorbance at $220 \mathrm{~nm}$. The molecular weight and purity of synthesized peptides were checked by MALDI-TOF mass spectrometry (Bruker Daltons) or electron spray LC/MS-2010 (Shimadzu) [42,43].

\section{Expression and purification of recombinant cystatins}

E. coli C41 (DE3), C43 (DE3) and RIL strains were transformed with plasmids containing the BrBmcys2b, BrBmcys2c, and JpIocys2a sequences, respectively. The recombinant proteins BrBmcys $2 \mathrm{~b}$ and BrBmcys2c were expressed in SOB medium with $0.4 \mathrm{mM}$ isopropyl- $\beta$-Dthiogalactopyrano (IPTG) for $16 \mathrm{~h}$ at $25^{\circ} \mathrm{C}$. rGSTJpIocys2a protein was expressed in LB medium with $0.1 \mathrm{mM}$ IPTG for $24 \mathrm{~h}$ at $37^{\circ} \mathrm{C}$. Cells were further harvested by centrifugation at $10,000 \times \mathrm{g}$ for $10 \mathrm{~min}$ at $4^{\circ} \mathrm{C}$. $\mathrm{rBrBm} c y s 2 \mathrm{~b}$ and $\mathrm{rBrBmcys} 2 \mathrm{c}$ were resuspended in phosphate buffer containing $100 \mathrm{mM}$ of imidazole (lysis buffer) and rGST-JpIocys2a in PBS. For cell lysis, the suspension was sonicated five times for $30 \mathrm{~s}$ at $40 \mathrm{MHz}$ on ice (Sonics or Qsonica, respectively). The soluble and insoluble fractions were separated by centrifugation at $10,000 \times \mathrm{g}$ for $10 \mathrm{~min}$ at $4{ }^{\circ} \mathrm{C}$ (Hitachi).

The soluble fractions containing the rBrBmcys $2 b$ and rBrBmcys2c were purified by nickel-chelating Sepharose chromatography (GE Healthcare). Briefly, the soluble fractions were filtered in $0.45-\mu \mathrm{m}$ filters (Whatman) and then applied into the columns previously equilibrated with phosphate lysis buffer. Proteins of interest were eluted with phosphate buffer containing $150 \mathrm{mM}$ of imidazole at room temperature. Eluted fractions were purified using centrifugal filter devices (Centricon YM10 - 50,000 MW cut-off, Millipore), lyophilized and dialyzed against PBS. The soluble fractions containing the rGST-JpIocys2a was purified by Glutathione Sepharose 4B (Amersham Bioscience) and eluted with $50 \mathrm{mM}$ Tris$\mathrm{HCl}$ buffer and $10 \mathrm{mM}$ glutathione, in $\mathrm{pH}$ 8.0. Purified rGST-JpIocys2a was dialyzed in cleavage buffer $(\mathrm{NaCl}$ $140 \mathrm{mM}, \mathrm{KCl} 2.7 \mathrm{mM}, \mathrm{Na}_{2} \mathrm{HPO}_{4} 10 \mathrm{mM}, \mathrm{KH}_{2} \mathrm{PO}_{4}$ $1.8 \mathrm{mM}, \mathrm{pH} 7.3)$ and cleaved by Thrombin $(0.01 \mathrm{u} / \mu \mathrm{g}$; Sigma). rGST fusion protein was bound by Glutathione Sepharose 4B, and pure rJpIocys $2 \mathrm{a}$ was recovered in the supernatant. Protein concentrations were determined using the BCA Protein Assay kit (Thermo Scientific) following the manufacturer's instructions.

\section{Enzymatic assays}

Remaining enzymatic activity and apparent dissociation constants (KIs) were estimated using different cathepsins (Sigma) to determine the inhibitory profile of $\mathrm{rBrBmcys} 2 \mathrm{~b}$, rBrBmcys2c, and rJplocys2a. Enzymes were preincubated with recombinant cystatins at different concentrations ranging from 10 to $500 \mathrm{nM}$ in the corresponding assay buffer for $15 \mathrm{~min}$, and protease-specific substrates (Sigma) were added to estimate residual enzyme activity. Enzymes concentrations are presented in Table 1. Assay buffer and enzymes were used as follows: $100 \mathrm{mM}$ sodium acetate, $\mathrm{pH} 5.5,100 \mathrm{mM} \mathrm{NaCl}, 1 \mathrm{mM}$ EDTA, and $0.005 \%$ TritonX-100 for bovine cathepsin $\mathrm{C}$ and human cathepsin L; $100 \mathrm{mM}$ sodium acetate, $\mathrm{pH} 5.5,60 \mathrm{mM} \mathrm{NaCl}, 1 \mathrm{mM}$ EDTA for bovine cathepsin B; 100 mM HEPES, pH 7.5, $1 \mathrm{mM}$ EDTA for human cathepsin G. For KIs determination, substrates were used as follows: Z-Phe-Arg-MCA (0.012-0.1 mM) for cathepsin L; Z-Arg-Arg-pNA (0.012$1.0 \mathrm{mM}$ ) for cathepsin B; Gly-Phe-pNA (0.6-2.25 mM) for cathepsin C; N-Succinyl-Ala-Ala-Pro-Phe-pNA (0.67 mM) for cathepsin G. Chromogenic (405 $\mathrm{nm}$ of absorption) and fluorescence intensity (370 and $460 \mathrm{~nm}$ for emission and excitation, respectively) assays were monitored in a microplate spectrophotometer (Spectramax Microplate Reader, Molecular Devices Corporation). Data were fit for appropriate tight-binding inhibitors using a nonlinear regression analysis equation (Morrison, 1969) performed using GraphPad Prism version 5.00 for Windows (GraphPad Software).

\section{Tick tissues extraction}

Salivary glands, ovary, gut, fat body and larvae were disrupted and homogenized using a mortar and pestle in an ice bath with $10 \mathrm{mM}$ phosphate buffer, $\mathrm{pH}$ 7.2. The homogenate was centrifuged at $16,000 \times \mathrm{g}$ for $15 \mathrm{~min}$ at $4^{\circ} \mathrm{C}$ to remove the insoluble material. Next, the soluble supernatant fraction was collected. The protein extracts were prepared according to the method previously described [44]. Tick hemolymph was collected as follows:

Table 1 Cystatins dissociation constants (KIs) for different proteases

\begin{tabular}{|c|c|c|c|c|c|}
\hline \multirow[t]{2}{*}{ Enzyme } & \multirow[t]{2}{*}{ Family } & \multirow{2}{*}{$\begin{array}{l}\text { Enzyme } \\
\text { concentration }\end{array}$} & \multicolumn{3}{|l|}{$\mathrm{Ki}(\mathrm{nM})$} \\
\hline & & & rBrBmcys2b & rBrBmcys2c & rJplocys2a \\
\hline Cathepsin B ${ }^{1}$ & Cysteine protease & $0.500 \mu \mathrm{M}$ & $0.82 \pm 0.35$ & n.i. & $154.70 \pm 106.71$ \\
\hline Cathepsin $C^{1}$ & Cysteine protease & $0.160 \mu \mathrm{M}$ & $26.65 \pm 6.75$ & $0.45 \pm 0.12$ & $>1 \mu \mathrm{M}$ \\
\hline Cathepsin L ${ }^{2}$ & Cysteine protease & $0.043 \mu \mathrm{M}$ & $2.48 \pm 1.00$ & $28.45 \pm 2.34$ & $4.39 \pm 1.76$ \\
\hline Cathepsin $\mathrm{G}^{2}$ & Serine protease & $0.100 \mu \mathrm{M}$ & n.i & n.i. & n.i. \\
\hline
\end{tabular}

n.i., not inhibited in the presence of $0.5 \mu \mathrm{M}$ recombinant cystatin.

${ }^{1}$ Bovine cathepsin.

${ }^{2}$ Human cathepsin. 
engorged $R$. microplus females were washed in alcohol $70 \%$, fixed to Petri dishes and kept at $4^{\circ} \mathrm{C}$; the cuticle was slit with a razor blade and the exuding hemolymph recovered and stored at $-20^{\circ} \mathrm{C}$. Saliva was collected by pilocarpine injection as previously described [45].

\section{Immunization of hamsters and rabbits}

Two hamsters were subcutaneously inoculated with $50 \mu \mathrm{g}$ of rBrBmcys2c or PBS. Immunizations consisted of four doses at 14-day intervals with the recombinant proteins emulsified in Freund's incomplete adjuvant. Two rabbits were subcutaneously immunized four times at 14-day intervals with $625 \mu \mathrm{g}$ of STQpep conjugated with carrier protein keyhole limpet hemocyanin (KLH; Sigma) or $200 \mu \mathrm{g}$ of GST from H. longicornis [46] both emulsified in Marcol-Moltanide (Exxon Mobil Corporation) adjuvant. Hamsters and rabbit blood were collected 14 days after the last booster, and sera were separated by centrifuging samples at $10,000 \times \mathrm{g}$ for $5 \mathrm{~min}$ at $4^{\circ} \mathrm{C}$. Serum aliquots were preserved at $-20^{\circ} \mathrm{C}$ upon use. Mice sera used in serological analysis were produced in a previous study [22].

\section{SDS-PAGE and Western blotting}

The production of recombinant cystatins and the presence of native cystatin in tissues were analyzed by SDSPAGE [47] and Western blot [48]. For Western blot analyses, recombinant cystatins (1 $\mu \mathrm{g}$ protein/lane), saliva (20 $\mu \mathrm{g}$ protein/lane), hemolymph (60 $\mu \mathrm{g}$ protein/ lane) and tissue extracts (150 $\mu \mathrm{g}$ protein/lane) were resolved in $14 \%$ gel for SDS-PAGE, followed by transference to nitrocellulose membranes. The membranes were blocked with 5\% non-fat dry milk in PBS and further incubated with mice, hamster or rabbit sera diluted to 1:50. Additionally, for rBmcys2b, rBmcys $2 \mathrm{c}$, and rJpIocys2a detection, anti-histidine tag or anti-GST-Hl antibodies $(1: 2,000)$ were used. After primarily sera incubations, anti-IgG species specific alkaline phosphatase (mouse and rabbit sera) and peroxidase (hamster serum) conjugates $(1: 5,000)$ were used as secondary antibodies. Alkaline phosphatase revelations were performed with NBT (nitro blue tetrazolium) and BCIP (5-bromo-4-chloro-3-indolyl phosphate, Sigma) in PBS. Peroxidase revelations were performed with DAB (3,3'-diaminobenzidine tetrahydrochloride), $\mathrm{H}_{2} \mathrm{O}_{2}$ and $\mathrm{CoCl}_{2}$ in PBS.

\section{Results}

\section{Jplocys2a identification and sequence analyses}

JpIocys2a nucleotide sequence showed an ORF of $423 \mathrm{bp}$, and the deduced amino acid sequence contained a signal peptide with cleavage site between amino acid residues 18 and 19 and four cysteine residues with a theoretical molecular weight of $15.6 \mathrm{kDa}$ (Figure 1). The G residue on $\mathrm{N}$-terminal, the motif $\mathrm{QxVxG}$ and the $\mathrm{PW}$ at the C-terminal are characteristic of family 2 cystatins, and are also conserved in JpIocys2a. The SND inhibitory domain, which inhibits legumain/asparaginyl endopeptidases found in human cystatin $\mathrm{C}$, is absent in JpIocys2a, like other tick cystatins [4].

\section{In silico antigenicity of cystatins and peptide selection}

The alignment of Jplocys2a with $R$. microplus cystatins showed conserved regions among the predicted amino acid sequences (Figure 2). The amino acid sequence STQVEGREYYDTVL from JpIocys2a (STQpep) was selected for peptide synthesis in accordance with the highest identity and antigenic region among all cystatins analyzed.

\section{Production of recombinant cystatins}

Soluble recombinant BrBmcys2b, BrBmcys2c, and GSTJpIocys2a were expressed in E. coli and purified by affinity chromatography. rGST-JpIocys2a was cleaved from GST-tag by thrombin and the purified rJplocys2a was recovered (Figure 3). SDS-PAGE showed that the conjugated rGST-JpIocys2a protein weight was about $42 \mathrm{kDa}$

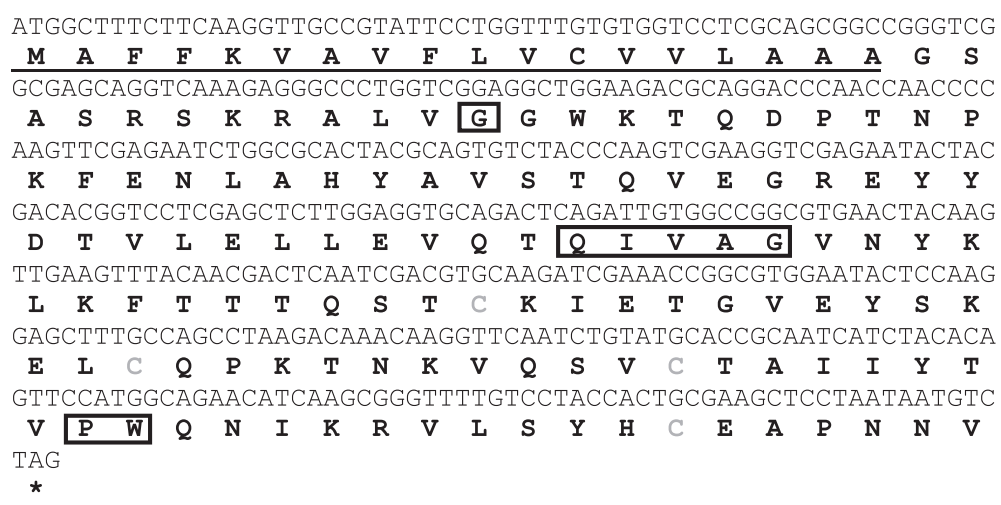

Figure 1 Jplocys2a nucleotide and predicted amino acid sequences. The predicted signal peptide (SignalP) is underlined. Cysteine residues are in gray and the conserved cystatin motifs PI (G), P II (QxVxG), and P III (PW) are boxed. 


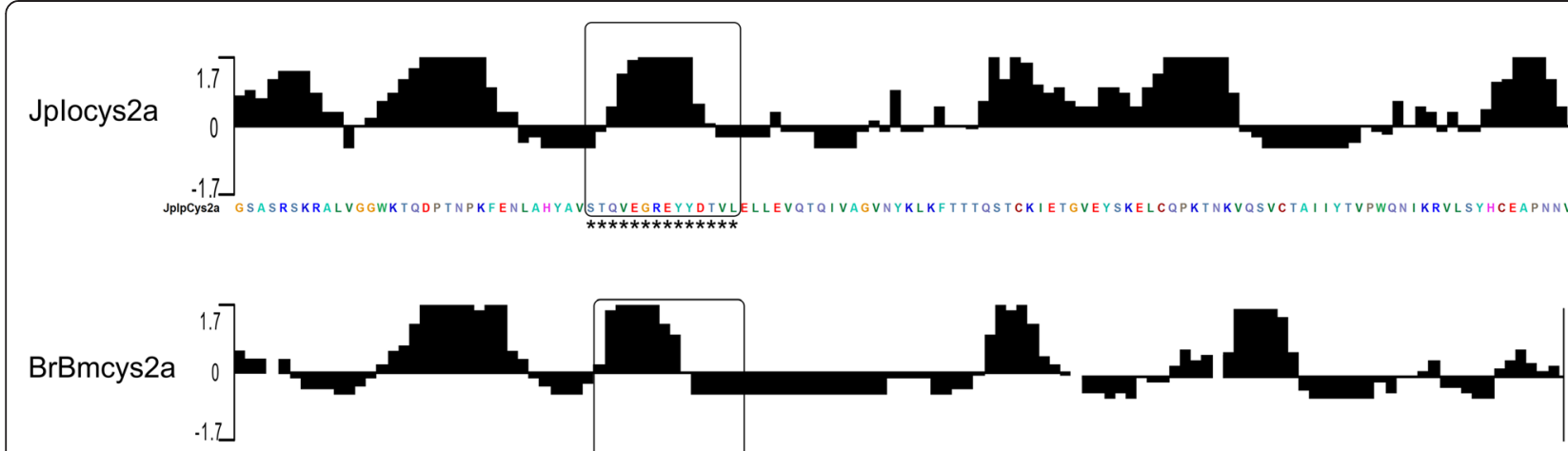

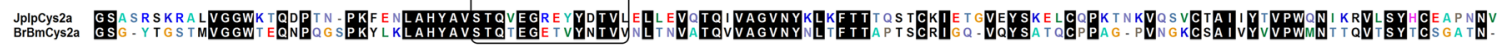
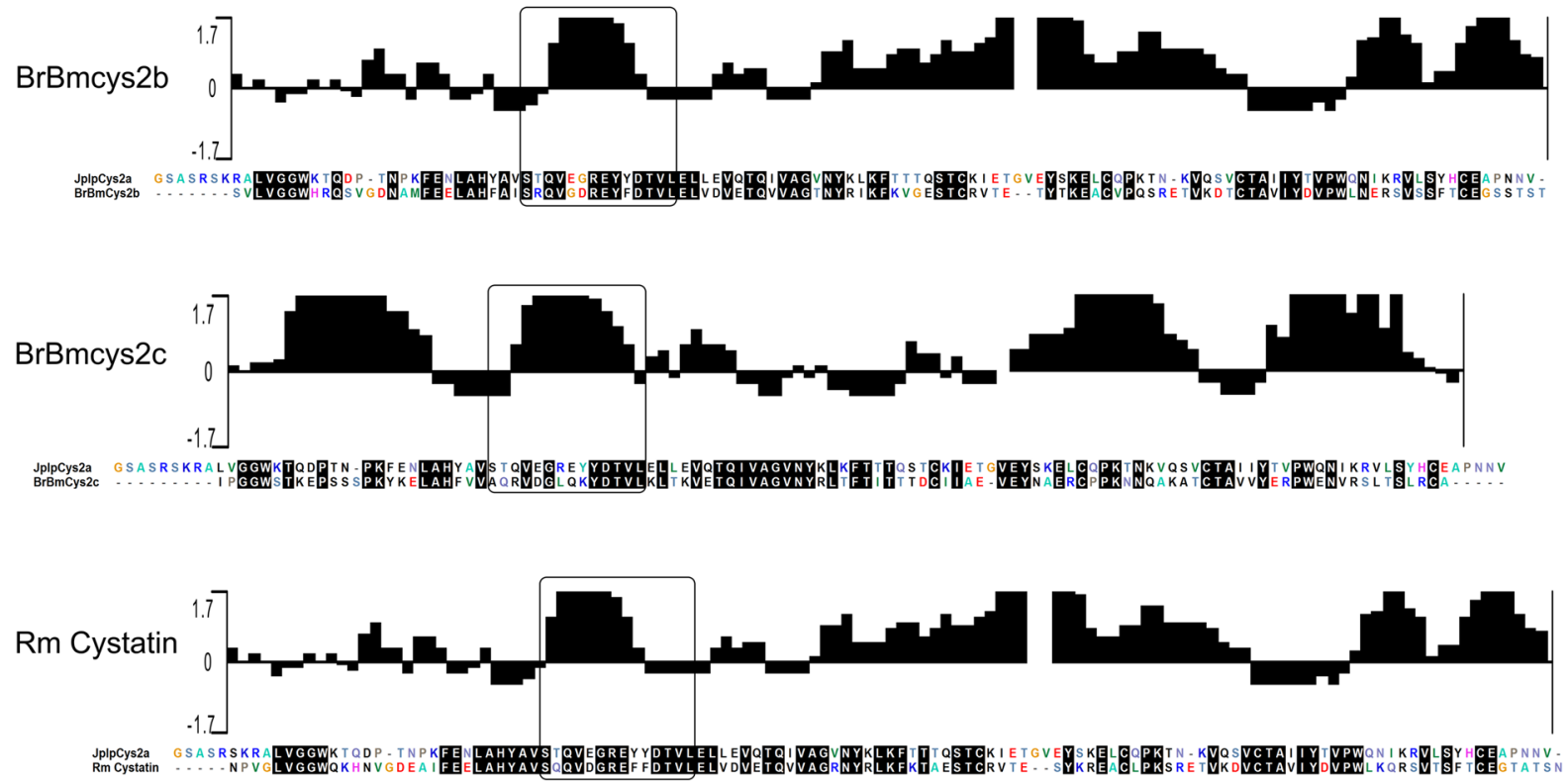

Figure 2 Conserved and antigenic tick cystatin regions for Jplocys 2 a peptide selection. Antigenic index plots for tick cystatins were predicted using the Jameson-Wolf algorithm. Graphic increased positivity shows predictive antigenic sites. Alignment shows conserved regions between Jplocys $2 a$ and $R$. microplus cystatins. Black boxes indicate conserved and antigenic amino acid region for each sequence. Asterisk indicate the selected region for peptide synthesis.

(30 kDa from rGST plus $12 \mathrm{kDa}$ from rJpIocys2a), while the weight of cystatins was approximately $12 \mathrm{kDa}$. These data are in accordance with in silico molecular weights estimation. rBrBmcys $2 b$ and $r B r B m c y s 2 c$ were recognized by anti-histidine tag antibodies, and rGSTJpIocys2a was recognized by anti-GST-Hl in Western blot assay. The purified recombinant cystatins were subsequently used in inhibitory and immunization assays.

rJplocys2a, rBrBmcys2b, and rBrBmcys2c inhibitory profile

Inhibitory assays were performed to characterize the specificity of rJplocys2a, rBrBmcys2b, and rBrBmcys2c for target enzymes (Figure 4 and Table 1). rJpIocys2a, rBrBmcys $2 \mathrm{~b}$, and $\mathrm{rBrBmcys} 2 \mathrm{c}$ modulated the activity of mammal cathepsins B, C, and $\mathrm{L}$ at distinct patterns. rJplocys2a and rBrBmcys $2 b$ inhibited all cysteine cathepsins, showing higher affinity for cathepsin $\mathrm{L}$ and $\mathrm{B}$, respectively (Table 1 ). In contrast, $\mathrm{rBrBm}$ cys $2 \mathrm{c}$ did not inhibit cathepsin $\mathrm{B}$, showing higher affinity for cathepsin C. Also, rJpIocys2a, rBrBmcys $2 \mathrm{~b}$, and $\mathrm{rBrBmcys} 2 \mathrm{c}$ inhibited these peptidases with apparent inhibition constants between 0.45 and 154.7 nM. The exception was rJplocys $2 \mathrm{a}$, which shows Ki higher than $1 \mu \mathrm{M}$ for cathepsin $\mathrm{C}$. Cathepsin $\mathrm{G}$, a serine protease, was not inhibited by these recombinant cystatins.

Recognition of native and recombinant cystatins by hyperimmune sera

Sera against STQpep, rBrBmcys2b and rBrBmcys2c were used to determine the presence of cystatins in $R$. 


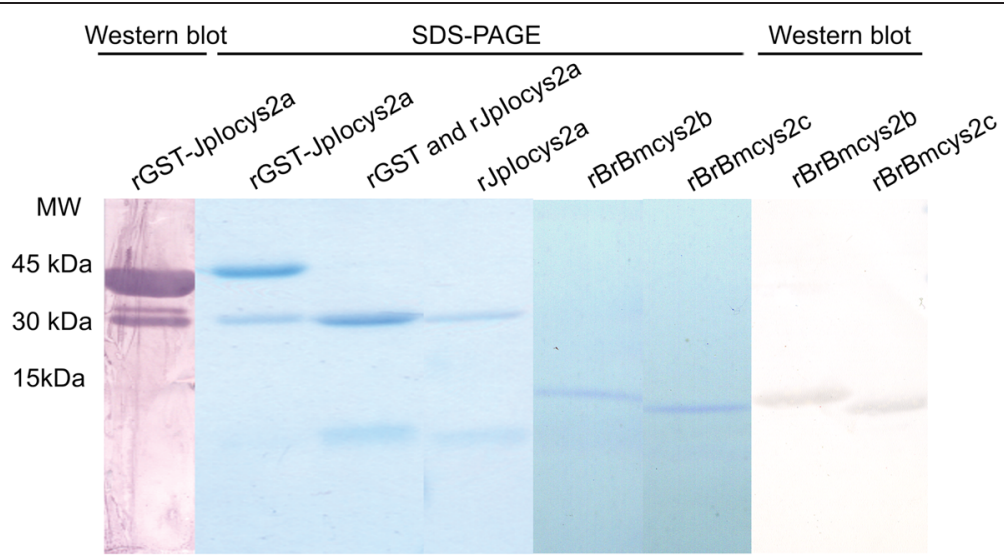

Figure 3 SDS-PAGE and Western blot of rJplocys2a, rBrBmcys2b and rBrBmcys2c production. Western blot: purified rGST-Jplocys2a probed with anti-GST-HI primary antibody and rabbit anti-lgG secondary antibody conjugate with alkaline phosphatase; purified rBrBmcys $2 \mathrm{~b}$ and rBrBmcys2c probed with anti-histidine tag primary antibody conjugate with alkaline phosphatase. Alkaline phosphatase revelations were performed with NBT and BCIP. SDS-PAGE: Recombinant cystatins resolved by 14\% SDS-PAGE were stained with Coomassie blue G-250; purified rGST-Jplocys2a before and after thrombin cleavage (rGST and Jplocys2a); purified rJplocys2a, rBrBmcys2b and rBrBmcys2c. MW: molecular weight.

microplus tissues as well as the cross-antigenicity between peptide, native, and recombinant cystatins (Figure 5). Native cystatins present in saliva, larvae, ovary, gut, salivary glands, and fat body (apparent molecular mass of $12 \mathrm{kDa}$ ) were differentially recognized by these sera. Native cystatins were recognized by anti-rBrBmcys $2 \mathrm{~b}$ in all tissues (Figure $5 \mathrm{~A}$ ), whereas anti-rBrBmcys2c sera recognized cystatins in gut from partially engorged females and in ovary, salivary glands and fat body from fully engorged females (Figure 5B). rBrBmcys2b and native cystatins from partially and fully engorged female salivary glands were recognized by anti-STQpep sera (Figure 5C). The hosts sera inoculated with PBS did not recognize native cystatins in these tissues (data not shown). Furthermore, antirBrBmcys2b serum detected cystatin in hemolymph (Figure 5A), unlike anti-rBrBmcys2c and negative controls sera. Since these sera were raised against STQpep and recombinant cystatins, this recognition shows the cross-antigenicity between native and peptide/recombinant cystatins.

\section{Phylogenetic analysis}

A neighbor-joining tree constructed with cystatin amino acid sequences from Ixodidae ticks is shown in Figure 6. We selected sequences of cystatins that have inhibitory profile and tissue localization that have been characterized. In the tree constructed, BrBmcys2b and BrBmcys2c grouped with other cystatins present in saliva/salivary glands and gut, respectively, showing tissue localization conservation between cystatin from the two branches of the tree. JpIocys2a grouped with the branch of cystatins expressed mainly in gut, suggesting the importance of this cystatin in tick blood digestion. All these cystatin are cathepsin L inhibitors, whereas cystatins that inhibit cathepsin B and $\mathrm{C}$ are present in the two branches.
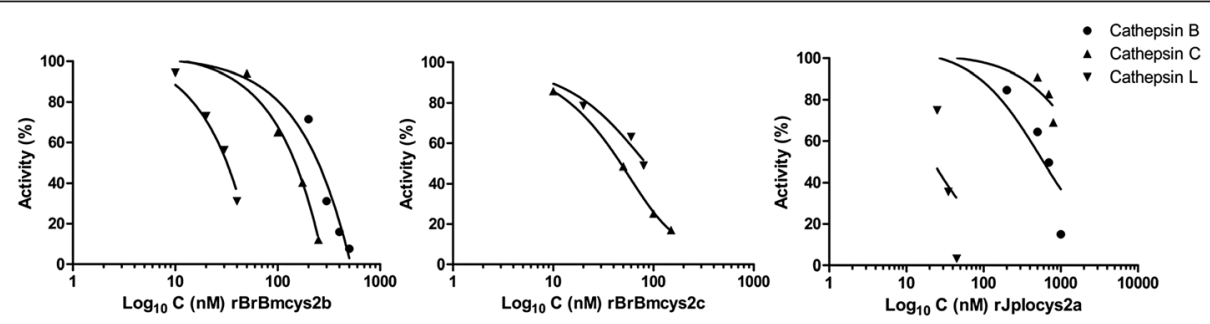

Figure 4 Activity inhibition assay of cathepsins $B, C$, and $L$ by rJplocys2a, rBrBmcys $2 b$, and rBrBmcys2c. Cathepsins $B, C$, and $L$ were incubated with Z-Arg-Arg-pNA (0.125 mM), Gly-Phe-pNA (1.8 mM), or Z-Phe-Arg-MCA (0.02 mM), respectively, in the presence of different concentrations of $r$.jplocys $2 \mathrm{a}, \mathrm{rBrBm}$ cys $2 \mathrm{~b}$, and rBrBmcys2c. The abscissa shows inhibitors concentration ( $\mathrm{nM}, \log _{10}$ ); the ordinate shows percentage of remaining enzymatic activity. Incubation of cathepsins B, C, and L without rJplocys2a, rBrBmcys $2 \mathrm{~b}$, and rBrBmcys2c represents $100 \%$ of enzyme activity. 


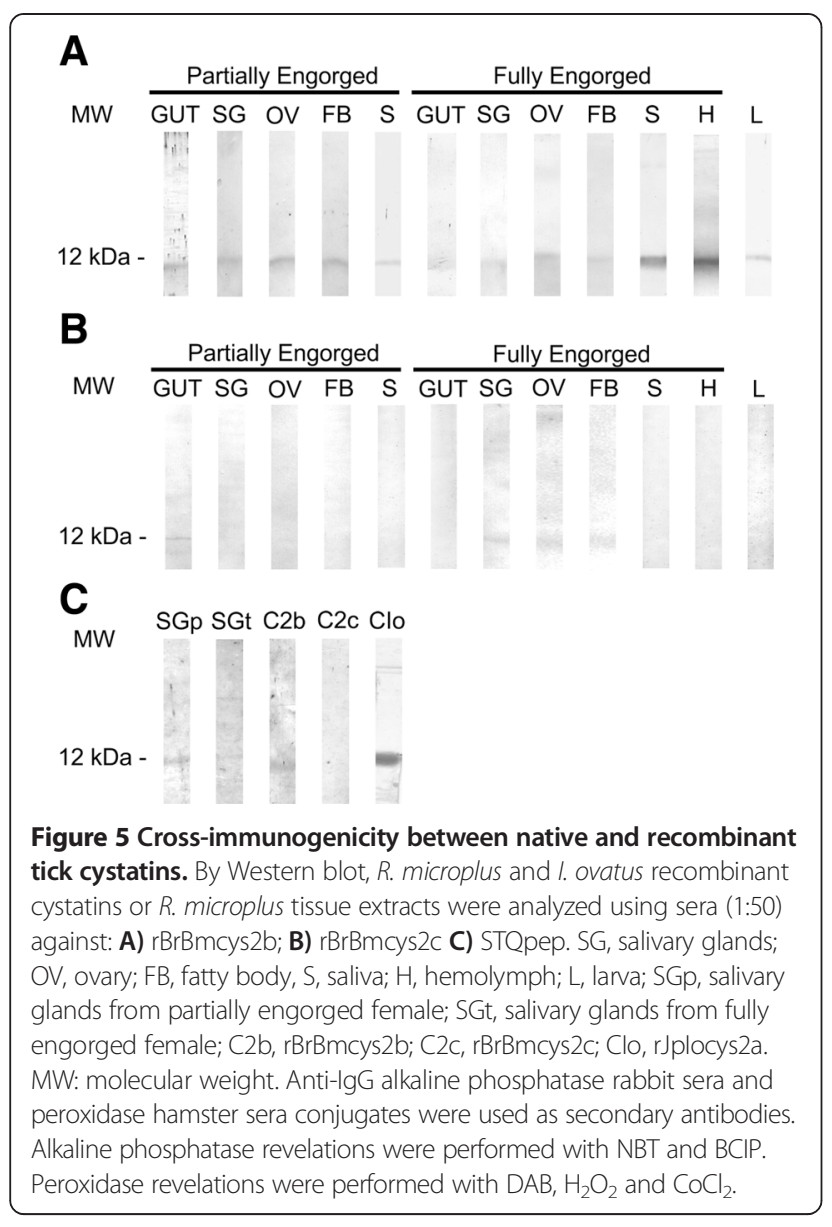

\section{Discussion}

The function of cystatins in parasite physiological processes is a subject of increasing interest among many research groups $[3,49]$. In ticks, however, only in recent years did cystatin roles begin to be elucidated [4]. The main goals of these works were the characterization of cathepsin targets, tissue localization and immune system modulation by tick cystatins. To date, no I. ovatus and only two $R$. microplus cystatin were biochemically characterized $[5,50]$. In the present work, we characterized the inhibition profile of one $I$. ovatus and two R. microplus cystatins. To uncover the inhibitory profile of these cystatins, we selected three cysteine proteases involved in host hemoglobin proteolytic degradation cascade by ticks [51-53]. In enzymatic inhibition assays, rBrBmcys2b inhibited cathepsins $\mathrm{B}, \mathrm{C}$, and $\mathrm{L}$ activities, and was detected in all tissues and secretions analyzed, suggesting the broad enzymatic regulation by this inhibitor in $R$. microplus physiology. Among the tissues from partially engorged females analyzed, BrBmcys2c was detected only in gut, suggesting the role of BrBmcys2c in blood metabolism. A potential target for this cystatin could be BmCL1, VTDCE and/or RmLCE, cathepsins from $R$. microplus that were detected in tick gut during feeding stages $[19,20,54]$. The pattern of native BrBmcys $2 \mathrm{~b}$ and BrBmcys2c expression corroborates previous qPCR results, which showed a higher mRNA transcripts expression in gut, as compared to other tick tissues [22]. The presence of BrBmcys2b in tissue that showed no transcription for this cystatin gene maybe the result of cystatin synthesis in gut, and its subsequent exportation to other tissues, similarly to other tick proteins $[21,55,56]$. The presence in hemolymph of BrBmcys2b, but not BrBmcys2c, supports this hypothesis. Jplocys2a inhibited cathepsins B, C and L at different levels. Cathepsin L and B were highly inhibited, when compared to $\mathrm{C}$ inhibition. The phylogenetic tree analysis show that JpIocys2a grouped together with gut cystatins, indicating that Jplocys2a could be secreted in I. ovatus gut lumen. In Ixodes ricinus blood digestion, cathepsins B and L hydrolyze hemoglobin secondary large fragments, whereas cathepsin $C$ degrades it down to small fragments [51]. Therefore, Jplocys2a may have major importance during modulation of initial hemoglobin degradation in blood digestion by ticks.

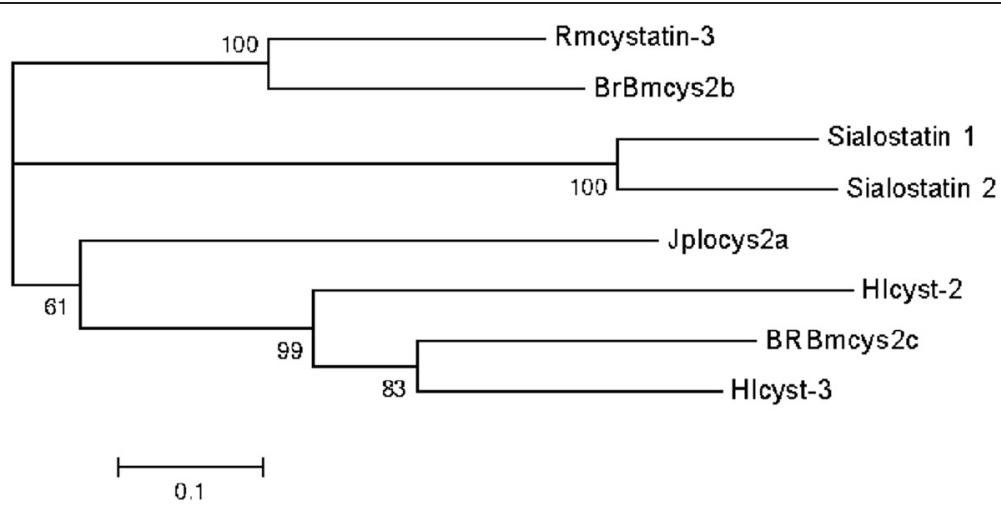

Figure 6 Phylogenetic analysis of tick cystatins. R. microplus BrBmcys2b [GenBank: KC816580], R. microplus BrBmcys2C [GenBank: KC816581], R. microplus Rmcystatin-3 [GenBank: AIX97454]; I. ovatus Jplocys2a [GenBank: KP253747]; I. scapularis sialostatin 1 [GenBank: AF483724], I. scapularis sialostatin 2 [GenBank: DQ066048]; H. longicornis Hlcyst-2 [GenBank: DQ364159], H. longicornis Hlcyst-3 [GenBank: EU426545]. Bootstrap values of 1,000 simulations are shown at the branches. 
In vitro and in vivo experiments have demonstrated the role of tick cystatins in the modulation of host immune system components responsible for anti-parasite infestation [10,16,57]. Similarly to Sialostatin L2 [37], rBrBmcys $2 b$ and $r B r B m c y s 2 c$ were not recognized by sera of tick-infested hosts (data not shown), indicating that the $R$. microplus cystatins are not immunogenic for bovines when inoculated through tick bite. The presence of BrBmcys2b in $R$. microplus saliva as well as its ability to inhibit cathepsin L suggests the participation of this cystatin in mechanisms to avoid host immune system. Cathepsin L is involved in mammal immune system process by MHC class II-presentation pathway regulation [58], as well as extracellular matrix breakdown during inflammation [59]. Cathepsin L secreted by macrophages inside tick feeding cavity would destroy host tissue elasticity, which is required for the effective parasite attachment [10]. Inhibition of cathepsin L by secreted tick cystatins would help tick feeding, whereas the absence of these compounds would result in inflammation and parasite rejection due to the host's immune response. This outcome was observed in tick cystatin knock-down and vaccination experiments [8,10,16,37]. Consequently, host cathepsin L inhibition would result in a weaker immune response against tick infestation. The presence of BrBmcys $2 b$ in partially and fully engorged female ovary indicated that this cystatin is important during egg development. In ovary, a vitellin degrading cysteine endopeptidase (VTDCE) that plays a crucial physiological role in egg maturation through vitellin mobilization $[21,54]$ is a potential enzyme target for BrBmcys2b.

In silico and in vitro comparative cross-antigenicity analyses of tick proteins, as CRTs [60], or GSTs [61], showed that, despite their high amino acid sequence conservation between homologues, these proteins display different immunodominant epitopes. Furthermore, antibodies against CRT and GST from $H$. longicornis were able to recognize native and recombinant forms of $R$. microplus homologous proteins [60,61]. Moreover, when GST-Hl was used in a vaccination trial against $R$. microplus infestation in cattle, the number of engorged females decreased by around 50\% [61]. To select immunodominant epitopes to explore the potential of cystatin in an anti-tick vaccine, a peptide from JpIocys2a was synthesized based on their antigenicity and similarity with $R$. microplus cystatins, and used for rabbit immunization. This approach seems to be interesting, because the immune response generated against designed peptide could be directed to immunodominant protective epitopes in native proteins. Synthetic peptide based vaccines have been studied as a strategy against malaria [62], hepatitis C [63,64], foot-and-mouth disease [65], human papilloma virus [66] and Toxoplasma gondii
[67]. Vaccination experiments using synthetic peptide from $R$. microplus Bm86 and Plasmodium falciparum also induced antibodies that recognized proteins from which the amino acid sequence had originated [68]. Furthermore, the peptides selected from $R$. microplus $\mathrm{Bm} 86$ developed a protection in cattle ranging between 36\% and $81 \%$ [68]. In this work, STQ-pep immunization generating antibodies that recognize native and recombinant $R$. microplus cystatins showed cross-reactivity in the selected peptide region. The results reveal that the peptide construct is immunogenic, allowing to recognize rJpIocys2a by anti-STQpep antibodies. The crossreaction of STQ-pep with native cystatins from $R$. microplus salivary glands and $\mathrm{rBrBmcys} 2 \mathrm{~b}$ paves the way for further works testing this peptide for a crossantigenic vaccine.

\section{Conclusions}

This work showed the differential presence of cystatins in $R$. microplus tick tissues, and demonstrates that cathepsins B, C, and L are modulated by JpIocys2a, BrBmcys2b, and BrBmcys2c to different degrees. These results suggest distinct tick physiological roles for these cystatins: BrBmcys $2 \mathrm{~b}$ acts during blood meal processing, egg and larva development, and in host immune system modulation; and BrBmcys2c and JpIocys2a act mainly during blood meal processing. Future anti-hemostatic and immunomodulatory experiments will better clarify the importance of JpIocys2a, BrBmcys2b, and BrBmcys2c in tick physiology.

\section{Competing interests}

The authors declare that they have no competing interests.

\section{Authors' contributions}

LFP, GAS, MFA, and AS carried out the experiments. LFP, GAS, CL, AM, and ISV designed the study. LFP, GAS, MFA, AS, CL, SK, KO, OM, and ISV drafted the manuscript. All authors read and approved the final manuscript.

\section{Acknowledgments}

This work was supported by grants from CNPq-Instituto Nacional de Ciência e Tecnologia de Entomologia Molecular, FINEP, CAPES, CNPq, FAPERJ and FAPERGS (Brazil), and MEXT (Japan). We would like to thank Dr. Carlos Termignoni and Msc. Lucas Tirloni for their assistance in enzymatic assays.

\section{Author details}

${ }^{1}$ Centro de Biotecnologia, Universidade Federal do Rio Grande do Sul, Avenida Bento Gonçalves, 9500, Prédio 43421, Porto Alegre 91501-970, RS, Brazil. 2Department of Disease Control, Laboratory of Infectious Diseases, Graduate School of Veterinary Medicine, Hokkaido University, 060-0818 Sapporo, Hokkaido, Japan. ${ }^{3}$ Departamento de Ciencias Microbiológicas, Laboratorio de Inmunología, Facultad de Veterinaria, UDELAR, Montevideo, Uruguay. ${ }^{4}$ Departamento de Farmacociências, Universidade Federal de Ciências da Saúde de Porto Alegre, Rua Sarmento Leite, 245, Porto Alegre 90050-170, RS, Brazil. ${ }^{5}$ Laboratório de Química e Função de Proteínas e Peptídeos-CBB-UENF and Unidade de Experimentação Animal, Avenida Alberto Lamego, 2000, Horto, Campos dos Goytacazes 28015-620, RJ, Brazil. 'Faculdade de Veterinária, Universidade Federal do Rio Grande do Sul, Avenida Bento Gonçalves, 9090, Porto Alegre 91540-000, RS, Brazil. ' Instituto Nacional de Ciência e Tecnologia em Entomologia Molecular, Rio de Janeiro, Brazil. 
Received: 17 December 2014 Accepted: 16 February 2015 Published online: 24 February 2015

\section{References}

1. Nicklin MJ, Barrett AJ. Inhibition of cysteine proteinases and dipeptidyl peptidasei by egg-white cystatin. Biochem J. 1984;223(1):245-53.

2. Zavasnik-Bergant T. Cystatin protease inhibitors and immune functions. Front Biosci. 2008;13:4625-37.

3. Hewitson JP, Grainger JR, Maizels RM. Helminth immunoregulation: the role of parasite secreted proteins in modulating host immunity. Mol Biochem Parasitol. 2009;167(1):1-11.

4. Schwarz A, Valdes JJ, Kotsyfakis M. The role of cystatins in tick physiology and blood feeding. Ticks Tick Borne Dis. 2012;3(3):117-27.

5. Lu S, Soares TS, Vaz IS, Lovato DV, Tanaka AS. Rmcystatin3, a cysteine protease inhibitor from Rhipicephalus microplus hemocytes involved in immune response. Biochimie. 2014;106C:17-23.

6. Renard G, Lara FA, de Cardoso FC, Miguens FC, Dansa-Petretski M, Termignoni C, et al. Expression and immunolocalization of a Boophilus microplus cathepsin L-like enzyme. Insect Mol Biol. 2002;11(4):325-8.

7. Yamaji K, Tsuji N, Miyoshi T, Islam MK, Hatta T, Alim MA, et al. A salivary cystatin, HISC-1, from the ixodid tick Haemaphysalis longicornis play roles in the blood-feeding processes. Parasitol Res. 2009;106(1):61-8.

8. Karim S, Miller NJ, Valenzuela J, Sauer JR, Mather TN. RNAi-mediated gene silencing to assess the role of synaptobrevin and cystatin in tick blood feeding. Biochem Biophys Res Commun. 2005;334(4):1336-42.

9. Kotsyfakis M, Sa-Nunes A, Francischetti IMB, Mather TN, Andersen JF, Ribeiro JMC. Antiinflammatory and immunosuppressive activity of sialostatin L, a salivary cystatin from the tick Ixodes scapularis. J Biol Chem. 2006; 281(36):26298-307.

10. Kotsyfakis M, Karim S, Andersen JF, Mather TN, Ribeiro JMC. Selective cysteine protease inhibition contributes to blood-feeding success of the tick Ixodes scapularis. J Biol Chem. 2007;282(40):29256-63.

11. Zhou J, Liao M, Ueda M, Gong H, Xuan X, Fujisaki K. Characterization of an intracellular cystatin homolog from the tick Haemaphysalis longicornis. Vet Parasitol. 2009;160(1-2):180-3.

12. Zhou J, Liao M, Gong H, Xuan X, Fujisaki K. Characterization of Hlcyst-3 as a member of cystatins from the tick Haemaphysalis longicornis. Exp Appl Acarol. 2010;51(4):327-33.

13. Zhou JL, Ueda M, Umemiya R, Battsetseg B, Boldbaatar D, Xuan XA, et al. A secreted cystatin from the tick Haemaphysalis longicornis and its distinct expression patterns in relation to innate immunity. Insect Biochem Mol Biol. 2006;36(7):527-35.

14. Yamaji K, Tsuji N, Miyoshi T, Hatta T, Alim MA, Anisuzzaman, et al. Hlcyst-1 and Hlcyst-2 are potential inhibitors of HICPL-A in the midgut of the Ixodid tick Haemaphysalis longicornis. J Vet Med Sci. 2010;72(5):599-604.

15. Grunclova L, Horn M, Vancova M, Sojka D, Franta Z, Mares M, et al. Two secreted cystatins of the soft tick Ornithodoros moubata: differential expression pattern and inhibitory specificity. Biol Chem. 2006;387(12):1635-44.

16. Salat J, Paesen GC, Rezacova P, Kotsyfakis M, Kovarova Z, Sanda M, et al. Crystal structure and functional characterization of an immunomodulatory salivary cystatin from the soft tick Ornithodoros moubata. Biochem J. 2010:429:103-12.

17. Imamura S, Konnai S, Yamada S, Parizi LF, Githaka N, Vaz ID, et al. Identification and partial characterization of a gut Rhipicephalus appendiculatus cystatin. Ticks Tick Borne Dis. 2013;4(1-2):138-44.

18. Grisi L, Leite RC, Martins JRD, de Barros ATM, Andreotti R, Cancado PHD, et al. Reassessment of the potential economic impact of cattle parasites in Brazil. Rev Bras Parasitol Vet. 2014;23(2):150-6.

19. Renard G, Garcia JF, Cardoso FC, Richter MF, Sakanari JA, Ozaki LS, et al. Cloning and functional expression of a Boophilus microplus cathepsin L-like enzyme. Insect Biochem Mol Biol. 2000;30(11):1017-26.

20. Estrela A, Seixas A, Termignoni C. A cysteine endopeptidase from tick (Rhipicephalus (Boophilus) microplus) larvae with vitellin digestion activity. Comp Biochem Physiol B Biochem Mol Biol. 2007;148(4):410-6.

21. Seixas A, Estrela AB, Ceolato JC, Pontes EG, Lara F, Gondim KC, et al. Localization and function of Rhipicephalus (Boophilus) microplus vitellindegrading cysteine endopeptidase. Parasitology. 2010;137(12):1819-31.

22. Parizi LF, Githaka NW, Acevedo C, Benavides U, Seixas A, Logullo C, et al. Sequence characterization and immunogenicity of cystatins from the cattle tick Rhipicephalus (Boophilus) microplus. Ticks Tick Borne Dis. 2013;4(6):492-9.
23. Robbins RG. The ticks (Acari : Ixodida : Argasidae, Ixodidae) of Taiwan: a synonymic checklist. Proc Entomol Soc Wash. 2005;107(2):245-53.

24. Chen Z, Yang XJ, Bu FJ, Yang XH, Yang XL, Liu JZ. Ticks (Acari: Ixodoidea: Argasidae, Ixodidae) of China. Exp Appl Acarol. 2010;51(4):393-404

25. Estrada-Pena A, Jongejan F. Ticks feeding on humans: a review of records on human-biting Ixodoidea with special reference to pathogen transmission. Exp Appl Acarol. 1999;23(9):685-715.

26. Murase Y, Konnai S, Githaka N, Hidano A, Taylor K, Ito T, et al. Prevalence of lyme borrelia in Ixodes persulcatus ticks from an area with a confirmed case of Lyme disease. J Vet Med Sci. 2013;75(2):215-8.

27. Chao LL, Liu LL, Ho TY, Shih CM. First detection and molecular identification of Borrelia garinii Spirochete from Ixodes ovatus tick ectoparasitized on stray cat in Taiwan. Plos One. 2014;9(10):e110599.

28. Takahashi Y, Sohnaka M, Nakao M, Miyamoto K, Fukunaga M. Characterization of Borrelia species isolated from Ixodid ticks, Ixodes ovatus. Microbiol Immunol. 1993;37(9):721-7.

29. Abbas RZ, Zaman MA, Colwell DD, Gilleard J, lqbal Z. Acaricide resistance in cattle ticks and approaches to its management: the state of play. Vet Parasitol. 2014;203(1-2):6-20.

30. Merino O, Alberdi P, Pérez de la Lastra JM, de la Fuente J. Tick vaccines and the control of tick-borne pathogens. Front Cell Infect Microbiol. 2013;3(30).

31. Parizi LF, Githaka NW, Logullo C, Konnai S, Masuda A, Ohashi K, et al. The quest for a universal vaccine against ticks: cross-immunity insights. Vet J. 2012;194(2):158-65.

32. Nijhof AM, Balk JA, Postigo M, Rhebergen AM, Taoufik A, Jongejan F. Bm86 homologues and novel ATAQ proteins with multiple epidermal growth factor (EGF)-like domains from hard and soft ticks. Int J Parasitol. 2010;40 (14):1587-97.

33. Agianian B, Tucker PA, Schouten A, Leonard K, Bullard B, Gros P. Structure of a Drosophila sigma class glutathione $S$-transferase reveals a novel active site topography suited for lipid peroxidation products. J Mol Biol. 2003;326(1):151-65.

34. Hajdusek O, Sojka D, Kopacek P, Buresova V, Franta Z, Sauman I, et al. Knockdown of proteins involved in iron metabolism limits tick reproduction and development. Proc Natl Acad Sci U S A. 2009;106(4):1033-8.

35. Havlikova S, Roller L, Koci J, Trimnell AR, Kazimirova M, Klempa B, et al. Functional role of 64P, the candidate transmission-blocking vaccine antigen from the tick, Rhipicephalus appendiculatus. Int J Parasitol. 2009;39(13):1485-94.

36. Galindo RC, Doncel-Perez E, Zivkovic Z, Naranjo V, Gortazar C, Mangold AJ, et al. Tick subolesin is an ortholog of the akirins described in insects and vertebrates. Dev Comp Immunol. 2009;33(4):612-7.

37. Kotsyfakis M, Anderson JM, Andersen JF, Calvo E, Francischetti IMB, Mather TN, et al. Cutting edge: immunity against a "Silent" salivary antigen of the Lyme vector Ixodes scapularis impairs its ability to feed. J Immunol. 2008; 181(8):5209-12.

38. Gonsioroski AV, Bezerra IA, Utiumi KU, Driemeier D, Farias SE, Vaz ID, et al. Anti-tick monoclonal antibody applied by artificial capillary feeding in Rhipicephalus (Boophilus) microplus females. Exp Parasitol. 2012;130(4):359-63.

39. Hall TA. BioEdit: a user-friendly biological sequence alignment editor and analysis program for windows 95/98/NT. Nucl Acids Symp Ser. 1999;41:95-8.

40. Wolf H, Modrow S, Motz M, Jameson BA, Hermann G, Fortsch B. An integrated family of amino acid sequence analysis programs. Comput Appl Biosci. 1988;4(1):187-91.

41. Tamura K, Peterson D, Peterson N, Stecher G, Nei M, Kumar S. MEGA5: Molecular Evolutionary Genetics Analysis using maximum likelihood, evolutionary distance, and maximum parsimony methods. Mol Biol Evol. 2011;28(10):2731-9.

42. Hirata IY, Cezari MHS, Nakaie CR, Boschcov P, Ito AS, Juliano MA, et al. Internally quenched luorogenic protease substrates: solid-phase synthesis and fluorescence spectroscopy of peptides containing ortho-aminobenzoyldinitrophenyl groups as donor-acceptor pairs. Lett Pept Sci. 1994;1:299-308.

43. Korkmaz B, Attucci S, Juliano MA, Kalupov T, Jourdan ML, Juliano L, et al. Measuring elastase, proteinase 3 and cathepsin $\mathrm{G}$ activities at the surface of human neutrophils with fluorescence resonance energy transfer substrates. Nat Protoc. 2008:3(6):991-1000.

44. Vaz ID, Logullo C, Sorgine M, Velloso FF, de Lima MFR, Gonzales JC, et al. Immunization of bovines with an aspartic proteinase precursor isolated from Boophilus microplus eggs. Vet Immunol Immunopathol. 1998;66(3-4):331-41.

45. Tirloni L, Reck J, Terra RMS, Martins JR, Mulenga A, Sherman NE, et al Proteomic analysis of cattle tick Rhipicephalus (Boophilus) microplus saliva: A comparison between partially and fully engorged females. Plos One. 2014;9(4):e94831. 
46. Vaz ID, Imamura S, Ohashi K, Onuma M. Cloning, expression and partial characterization of a Haemaphysalis longicornis and a Rhipicephalus appendiculatus glutathione S-transferase. Insect Mol Biol. 2004;13(3):329-35.

47. Laemmli UK. Cleavage of structural proteins during assembly of head of Bacteriophage-T4. Nature. 1970;227(5259):680-5.

48. Johnson DA, Gautsch JW, Sportsman JR, Elder JH. Improved technique utilizing nonfat dry milk for analysis of proteins and nucleic acids transferred to nitrocellulose. Gene Anal Tech. 1984;1(1):3-8.

49. Hartmann S, Lucius R. Modulation of host immune responses by nematode cystatins. Int J Parasitol. 2003;33(11):1291-302.

50. Lima CA, Sasaki SD, Tanaka AS. Bmcystatin, a cysteine proteinase inhibitor characterized from the tick Boophilus microplus. Biochem Biophys Res Commun. 2006;347(1):44-50.

51. Horn M, Nussbaumerova M, Sanda M, Kovarova Z, Srba J, Franta Z, et al. Hemoglobin digestion in blood-feeding ticks: mapping a multipeptidase pathway by functional proteomics. Chem Biol. 2009;16(10):1053-63.

52. Franta Z, Frantova H, Konvickova J, Horn M, Sojka D, Mares M, et al. Dynamics of digestive proteolytic system during blood feeding of the hard tick Ixodes ricinus. Parasit Vectors. 2010;3:119.

53. Sojka D, Franta Z, Horn M, Caffrey CR, Mares M, Kopacek P. New insights into the machinery of blood digestion by ticks. Trends Parasitol. 2013; 29(6):276-85.

54. Seixas A, Dos Santos PC, Velloso FF, Vaz ID, Masuda A, Horn F, et al. A Boophilus microplus vitellin-degrading cysteine endopeptidase. Parasitology. 2003:126:155-63.

55. Pohl PC, Sorgine MHF, Leal AT, Logullo C, Oliveira PL, Vaz ID, et al. An extraovarian aspartic protease accumulated in tick oocytes with vitellin-degradation activity. Comp Biochem Physiol B Biochem Mol Biol. 2008;151(4):392-9

56. Galay RL, Aung KM, Umemiya-Shirafuji R, Maeda H, Matsuo T, Kawaguchi H, et al. Multiple ferritins are vital to successful blood feeding and reproduction of the hard tick Haemaphysalis longicornis. J Exp Biol. 2013;216(10):1905-15.

57. Kotsyfakis M, Horka H, Salat J, Andersen JF. The crystal structures of two salivary cystatins from the tick Ixodes scapularis and the effect of these inhibitors on the establishment of Borrelia burgdorferi infection in a murine model. Mol Microbiol. 2010;77(2):456-70.

58. Hsing LC, Rudensky AY. The lysosomal cysteine proteases in MHC class II antigen presentation. Immunol Rev. 2005;207:229-41.

59. Serveau-Avesque C, Ferrer-Di Martino M, Herve-Grepinet V, Hazouard E, Gauthier F, Diot $E$, et al. Active cathepsins B, HK, L and S in human inflammatory broncho-alveolar lavage fluids. Biol Cell. 2006;98(1):15-22.

60. Parizi LF, Rech H, Ferreira CAS, Imamura S, Ohashi K, Onuma M, et al. Comparative immunogenicity of Haemaphysalis longicornis and Rhipicephalus (Boophilus) microplus calreticulins. Vet Parasitol. 2009;164(2-4):282-90.

61. Parizi LF, Utiumi KU, Imamura S, Onuma M, Ohashi K, Masuda A, et al. Cross immunity with Haemaphysalis longicornis glutathione S-transferase reduces an experimental Rhipicephalus (Boophilus) microplus infestation. Exp Parasitol. 2011;127(1):113-8

62. Sirima SB, Tiono AB, Ouedraogo A, Diarra A, Ouedraogo AL, Yaro JB, et al. Safety and immunogenicity of the malaria vaccine candidate MSP3 long synthetic peptide in 12-24 months-old burkinabe children. Plos One. 2009; 4(10):e7549

63. El-Awady MK, Tabll AA, El-Abd YS, Yousif H, Hegab M, Reda M, et al. Conserved peptides within the $E 2$ region of Hepatitis $C$ virus induce humoral and cellular responses in goats. Virol J. 2009;6(66)

64. El-Awady MK, Tabll AA, Yousif H, El-Abd Y, Reda M, Khalil SB, et al. Murine neutralizing antibody response and toxicity to synthetic peptides derived from E1 and E2 proteins of hepatitis C virus. Vaccine. 2010;28(52):8338-44.

65. Tang H, Liu X, Fang Y, Jiang S, Pan L, LV J, et al. Evaluation of synthetic peptide vaccines against foot-and-mouth disease type A. Appl Microbiol Biotechnol. in press.

66. van Poelgeest MIE, Welters MJP, van Esch EMG, Stynenbosch LFM, Kerpershoek G, van Meerten ELV, et al. HPV16 synthetic long peptide (HPV16-SLP) vaccination therapy of patients with advanced or recurrent HPV16-induced gynecological carcinoma, a phase II trial. J Transl Med. 2013;11(88).

67. Wang Y, Wang G, Ou J, Yin H, Zhang D. Analyzing and identifying novel B cell epitopes within Toxoplasma gondii GRA4. Parasit Vectors. 2014;7(1):474

68. Patarroyo JH, Portela RW, De Castro RO, Pimentel JC, Guzman F, Patarroyo $M E$, et al. Immunization of cattle with synthetic peptides derived from the Boophilus microplus gut protein (Bm86). Vet Immunol Immunopathol. 2002;88(3-4):163-72

\section{Submit your next manuscript to BioMed Central and take full advantage of:}

- Convenient online submission

- Thorough peer review

- No space constraints or color figure charges

- Immediate publication on acceptance

- Inclusion in PubMed, CAS, Scopus and Google Scholar

- Research which is freely available for redistribution 